\title{
Search for a Lorentz invariant velocity distribution of a relativistic gas
}

\author{
Evaldo M. F. Curado ${ }^{1,2}$, Felipe T. L. Germani ${ }^{1}$ \\ and Ivano Damião Soares ${ }^{1}$ \\ ${ }^{1}$ Centro Brasileiro de Pesquisas Físicas - CBPF \\ ${ }^{2}$ National Institute of Science and Technology \\ for Complex Systems \\ Rio de Janeiro, Brazil
}

\begin{abstract}
We examine the problem of the relativistic velocity distribution in a 1-dim relativistic gas in thermal equilibrium. We use numerical simulations of the relativistic molecular dynamics for a gas with two components, light and heavy particles. However in order to obtain the numerical data our treatment distinguishes two approaches in the construction of the histograms for the same relativistic molecular dynamic simulations. The first, largely considered in the literature, consists in constructing histograms with constant bins in the velocity variable and the second consists in constructing histograms with constant bins in the rapidity variable which yields Lorentz invariant histograms, contrary to the first approach. For histograms with constant bins in the velocity variable the numerical data are fitted accurately by the Jüttner distribution which is also not Lorentz invariant. On the other hand, the numerical data obtained from histograms constructed with constant bins in the rapidity variable, which are Lorentz invariant, are accurately fitted by a Lorentz invariant distribution whose derivation is discussed in this paper. The histograms thus constructed are not fitted by the Jütter distribution (as they should not). Our derivation is based on the special theory of relativity, the central limit theorem and the Lobachevsky structure of the velocity space of the theory, where the rapidity variable plays a crucial
\end{abstract}


role. For $v^{2} / c^{2} \ll 1$ and $1 / \beta \equiv k_{B} T / m_{0} c^{2} \ll 1$ the distribution tends to the Maxwell-Boltzmann distribution.

\section{Introduction and summary of results}

In physics, it is difficult to overestimate adequately the importance of the Maxwell-Boltzmann (MB) distribution of velocities for gases, introduced by Maxwell in 1860 [1]. It was the first time that a probability concept was introduced in a physical theory, as the existing theories at the time were purely deterministic like Newtonian mechanics and wave theory. Actually the work of Maxwell was the starting point for Boltzmann to elaborate his research program on the evolution of a time dependent distribution of velocities for gases, culminating in the articles of 1872 [2] and 1877[3], among other important papers, which are amid the fundamental cornerstones of the modern kinetic theory of gases and of statistical mechanics.

With the implicit use of the atomic theory of matter (at that time a controversial theory), the new concept of entropy was established having also as its starting point the introduction, by Maxwell, of the concept of probability. Since then the Maxwell-Boltzmann (MB) distribution played a fundamental role in the statistical description of gaseous systems with a large number of constituents. Actually, in many cases it is considerably simpler, and even as accurate as, to use the MB distribution instead of Bose-Einstein and Fermi-Dirac distributions [4. However a clear limitation of the $\mathrm{MB}$ distribution is its nonrelativistic character, encompassing velocities larger than the velocity of light in contradiction with the special theory of relativity.

In the present paper we introduce a 1- dim Lorentz invariant (LI) distribution of velocities for a relativistic gas in thermal equilibrium which has the MB distribution as a limit for velocities much smaller than the velocity of light (with correspondingly relatively small temperatures). A discussion about this distribution in the 1-dim and 3 -dim cases was given by two of us in [5]. Our derivation was based on three pillars: the special theory of relativity, the central limit theorem and the fact that the velocity space of special relativity is a Lobachevsky space, where the additivity of velocities in the Galilean relativity is transferred to the additivity of rapidities.

We have made a numerical simulation of the relativistic dynamics for a 1-dim relativistic molecular gas with two components (light and 

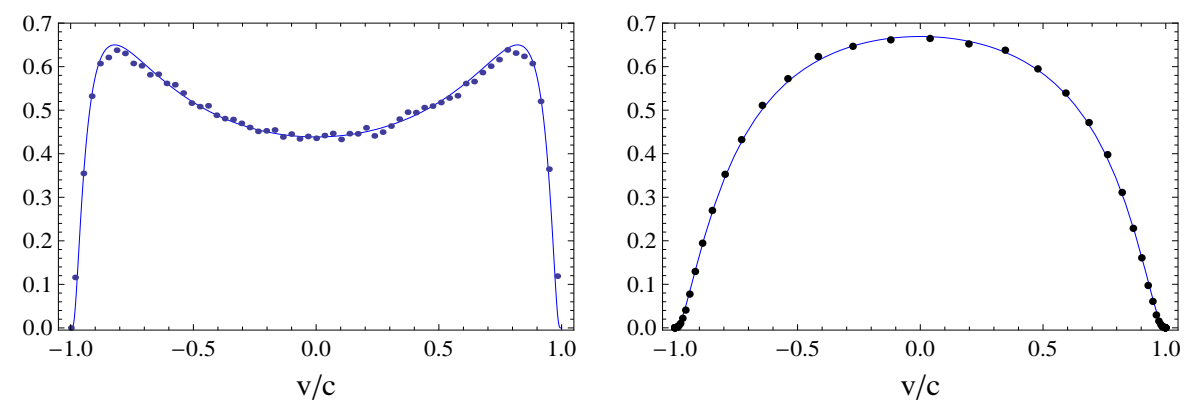

Figure 1: Plot of the equilibrium velocity distributions of heavy particles $\left(N_{2}=2,500\right)$ obtained from the same numerical simulation for the relativistic molecular dynamics of a 1-dim gas with $N=5,000$ particles: (left) the points are obtained from a histogram of constant velocity bins, which is not LI, and the continuous curve is the best fit of the points to the Jüttner distribution (9), with best fit parameter $m_{0} \beta_{J} \simeq 0.8570$ ( $\mathrm{rms}$ error $\simeq 0.017$ ). (right) The points are obtained from a histogram of constant rapidity bins, which is LI, transformed into a normalized histogram in the velocities (via eqs. (10) and (11)) with non constant bins but still LI. The continuous curve is the best fit of the points to the LI velocity distribution (8) with best fit parameter $\beta \simeq 1.4070$ (rms error $\simeq 0.004$ ). 
heavy particles) in the same vein of Cubero et al. [6]. We used $N_{1}=$ 2,500 light particles of mass $m_{0}$ and $N_{2}=2,500$ heavy particles of mass $2 m_{0}$, with a total of 100 relativistic simulations (taking the box as the frame of reference in the simulations), and collected the data fixing the number 60 of points to be obtained in the histograms. The initial random distributions of the velocity of the particles must satisfy $-1<v_{i} / c<1$ with $\sum_{i} v_{i}=0$ (so that the box has zero velocity). The control of how much relativistic is the system is made by how the interval of the initial random distributions is closer to 1: the random choice of $\left|v_{i} / c\right|$ in the interval $[0.75,1)$ is considered less relativistic than in the interval $[0.85,1)$. The four-momentum $P_{a}$ of the particles is conserved for the whole relativistic evolution, with the relative speed of the colliding particles being a constant in the collision. The numerical methods follow closely [7, 8, 9].

To proceed, for the same relativistic simulations we adopted two distinct approaches in the construction of the histograms of the distribution of velocities in the thermalization limit: the first, histograms with constant velocity bins (which are not LI) and the second, histograms with constant rapidity bins (which are LI). In the second approach, from the constant rapidity histograms we obtain histograms of the velocities by using the inverse transformations of Eqs. (11) and (10). These transformations change the relative size of the bins, with the bins no longer equally spaced in a velocity scale as they were in a rapidity scale. However these histograms still remain LI.

It is crucial to remark here that the use of the rapidity is not merely a change of variables. The core of our new approach in this paper is that we treat the numerical data obtained from the molecular dynamics simulations by constructing the histograms with constant bins in the rapidity which is a LI procedure. This yields a LI probability distribution that has a completely distinct measure from previous treatments in the literature (cf. [6]) where the construction of histograms is made with constant bins in the velocity, a procedure that is clearly not LI and results in a completely distinct distribution. The distribution made with constant bins in the velocity cannot be transformed into the LI distribution obtained in this paper by a change of variables.

Part of our main results are displayed in Figs. 1 and 2 where we show the equilibrium velocity distributions derived from our numerical simulations for the light and heavy components of the 1-dim relativistic gas, respectively. For the same simulation, the points are obtained 

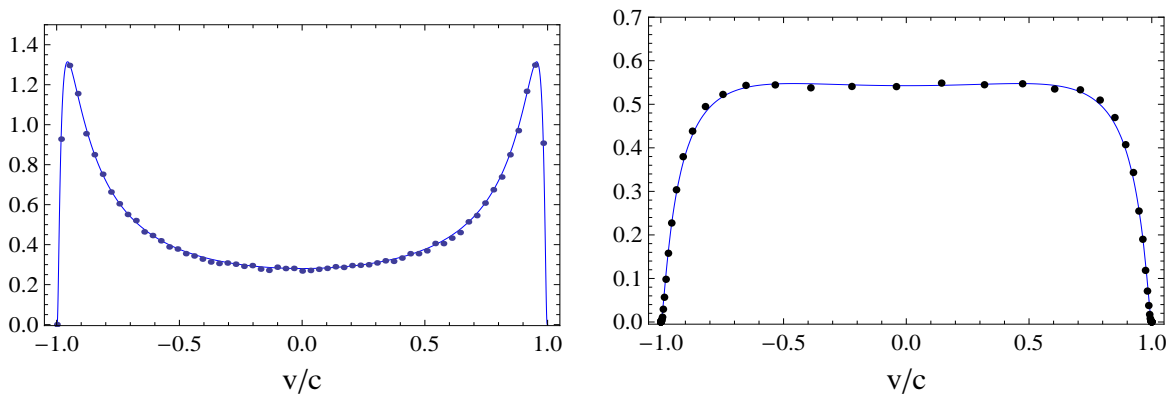

Figure 2: Plot of the equilibrium velocity distributions of light particles $\left(N_{1}=2,500\right)$ corresponding to the same numerical simulation of Figs. 1; (left) the points are obtained from a histogram with constant velocity bins, which is not LI, and the continuous curve is the best fit of the points to the Jüttner distribution (9), with best fit parameter $m_{0} \beta_{J} \simeq 0.8862(\mathrm{rms}$ error $\simeq 0.009$ ). (right) The points are obtained from a histogram of constant rapidity bins, which is LI, transformed into a normalized histogram in the velocities (via eqs. (10) and (11)) with non constant bins but still LI. The continuous curve is the best fit of the points to the Lorentz invariant velocity distribution (8) with best fit parameter $\beta \simeq 0.9254$ ( $\mathrm{rms}$ error $\simeq 0.008$ ).

either from a histogram of constant velocity bins (left figures), which is not LI, or from a histogram of constant rapidity bins (right figures) which is LI.

In Figs. 1 and 2 (left), where histograms of constant velocity bins are adopted, the continuous curves correspond to the best fit of the Jüttner distribution (9) [10]. The accurate fits reproduce a result of Cubero et al. [6] which is a standard reference in the literature on the subject, being considered as a clear evidence that Jüttner's is the correct relativistic distribution although not being a LI distribution. With the same procedure this result was later also verified for the case of 2-dim [11, 12] and 3-dim[13] distributions.

In Figs. 1 and 2 (right), where histograms of constant rapidity bins are adopted (properly transformed to a histogram where the abscissa is the difference of velocities), the continuous curves correspond to the best fit of the LI velocity distribution (8).

These two contrasting results, derived from the same numerical simulations, are connected to the distinct particular choices made on constructing histograms, namely, whether we choose the histogram to 


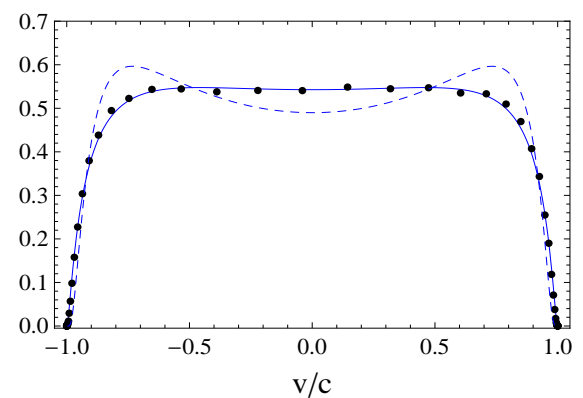

Figure 3: Plot of the velocity distribution of Figs. 2 (right): the dashed curve is the best fit of the points to the Jüttner distribution (9), with best fit parameter $m_{0} \beta_{J} \simeq 2.0406$ ( rms error $\left.\simeq 0.04\right)$. We see that while the LI velocity distribution fits quite accurately the points, the Jüttner distribution cannot be a candidate for a LI distribution.

be LI or not, a fact that has not yet been taken into account in the literature.

On the other hand the graphs of Fig. 3, done for light particles, show that the points obtained from a histogram of constant rapidity bins (which is LI) fit quite well the LI distribution (8) (continuous line) and give evidence that the Jüttner distribution (9) cannot be a candidate for a LI velocity distribution (dashed line).

\section{A proposal for a 1-dim LI velocity distribution}

We start by presenting a derivation of the $\mathrm{MB}$ velocity distribution which differs from the derivation used by Maxwell but which will be more appropriate in our derivation of a LI velocity distribution. Let us first consider the addition of velocities in the Galilean space. We know that the velocities add according the rule $\mathbf{v}=\sum_{i} \mathbf{v}_{i}$. Assuming that the velocities of the particles $\mathbf{v}_{i}$ are random variables, with zero mean, and considering that the sum is over a very large number of particles, we have - by the central limit theorem [14] - that the probability distribution of velocities for the random variable $\nu \equiv(1 / \sqrt{N}) \sum_{i} \mathbf{v}_{i}$ approaches the distribution $P(\nu) \propto \exp \left(-b \nu^{2}\right)$ or $P(\mathbf{v}) \propto \exp \left(-b \mathbf{v}^{2} / N\right)$, recovering thus the famous $\mathrm{MB}$ distribution if we plot $\sqrt{N} P(\mathbf{v})$ versus 
$\mathbf{v} / \sqrt{\mathbf{N}}$.

As well known, in the special theory of relativity the relative velocity $\mathbf{v}$ of two particles with arbitrary velocities $\mathbf{v}_{\mathbf{1}}$ and $\mathbf{v}_{\mathbf{2}}$, with respect to a fixed inertial frame, is given by

$$
\mathbf{v}=\frac{\mathbf{v}_{\mathbf{1}}-\mathbf{v}_{\mathbf{2}}+\left(\gamma\left(v_{2}\right)-1\right)\left(\mathbf{v}_{\mathbf{2}} / v_{2}^{2}\right)\left[\mathbf{v}_{\mathbf{1}} \cdot \mathbf{v}_{\mathbf{2}}-v_{2}^{2}\right]}{\gamma\left(v_{2}\right)\left(1-\mathbf{v}_{\mathbf{1}} \cdot \mathbf{v}_{\mathbf{2}} / c^{2}\right)}
$$

The above expression also holds with the interchange $\mathbf{v}_{\mathbf{1}} \leftrightarrow \mathbf{v}_{\mathbf{2}}$. The square of the modulus of the relative velocity is given by

$$
v^{2}=\frac{\left(\mathbf{v}_{\mathbf{1}}-\mathbf{v}_{\mathbf{2}}\right)^{2}-\left(1 / c^{2}\right)\left[\mathbf{v}_{\mathbf{1}} \wedge \mathbf{v}_{\mathbf{2}}\right]^{2}}{\left(1-\mathbf{v}_{\mathbf{1}} \cdot \mathbf{v}_{\mathbf{2}} / c^{2}\right)^{2}},
$$

which is symmetric with respect to $\mathbf{v}_{\mathbf{1}}$ and $\mathbf{v}_{\mathbf{2}}$. It is also a well known fact that the square of the relative velocity (2) is invariant under Lorentz transformations [15]. Let us now consider a fixed inertial reference frame, say the laboratory frame. For simplicity, we will assume one dimensional only. Let the velocity of two particles be $v_{1}$ and $v_{2}$, as measured in this inertial frame. The relative velocity of the two particles is given from (2) as

$$
v=\frac{v_{1}+v_{2}}{1+v_{1} v_{2} / c^{2}}
$$

which is invariant under Lorentz transformations. However (3) can be rewritten as

$$
\frac{1+v / c}{1-v / c}=\left(\frac{1+v_{1} / c}{1-v_{1} / c}\right)\left(\frac{1+v_{2} / c}{1-v_{2} / c}\right)
$$

and can be extended to any number of particles,

$$
\frac{1+v / c}{1-v / c}=\prod_{i}\left(\frac{1+v_{i} / c}{1-v_{i} / c}\right) .
$$

Taking the logarithm on both sides of Eq. (5) and defining

$$
\sigma_{i} \equiv \frac{1}{2} \ln \left(\frac{1+v_{i} / c}{1-v_{i} / c}\right)=\tanh ^{-1}\left(v_{i} / c\right), \quad \sigma_{i} \in(-\infty, \infty),
$$

we can express (5) as

$$
\sigma=\sum_{i} \sigma_{i}
$$


The variable defined in (6) is the rapidity associated with the velocity $v_{i}$ and will play a fundamental role in the remaining of the paper. Accordingly the rapidity of relatives velocities are therefore quantities which are LI and are additive in the arithmetic sense.

Let us then consider a relativistic gas with a large number of particles, each with a velocity $v_{i}$ as measured with respect to the inertial frame of the laboratory 1 . We concur that any Boltzmann-type equation (relativistic or not) that give rise to a universal stationary velocity distribution implicitly assumes the presence of a spatial confinement, thus singling out a preferred frame (cf. Cubero et al. [6] and references therein). However as we will see the LI distribution function derived here will be independent of such a singling out of frame, as the variables involved are LI. If we assume that the velocities $v_{i}$ are independent and random variables, with zero mean, the variables $\sigma_{i}$ 's are also independent and random with zero mean, and we have - in accordance with the central limit theorem - that the probability distribution for the variable $s \equiv \sigma / \sqrt{N}$ in an interval $s$ and $s+d s$ approaches $P(s) d s=C_{1} e^{-\tilde{\beta} s^{2}} d s$ or, denoting $\beta \equiv \tilde{\beta} / N$, $P(\sigma) d \sigma=C_{1} e^{-\beta \sigma^{2}} d \sigma / \sqrt{N}$, where $C_{1}=\sqrt{\tilde{\beta} / \pi}$ is a normalization constant. Using (6) and that $d \sigma=\gamma^{2} d v / c$, where $\gamma=1 / \sqrt{1-v^{2} / c^{2}}$, we can write the probability distribution for the velocities of a 1-dim relativistic gas as

$$
P(v) d v=C_{1} e^{-\beta\left(\tanh ^{-1}(v / c)\right)^{2}} \gamma^{2} d v / c \sqrt{N} .
$$

The factor $\gamma^{2} d v$ in (8) is the LI 1-dim line element. The adimensional parameter $\beta=m_{0} c^{2} / \kappa_{B} T$, where $\kappa_{B}$ is the Boltzmann constant, decreases with the temperature $T$. Such temperature is obviously LI. The distribution (8) is a 1-dim LI velocity distribution for a relativistic gas. In Fig. 4 we plot the distribution (8) for a fixed mass and several decreasing parameters $\beta$.

The LI velocity distribution changes the concavity at zero velocity for the particular value $\beta=1$ which separates highly relativistic domains from domains that contain the Maxwell-Boltzmann limit. Above $\beta=1$ the zero velocity concavity is negative; below $\beta=1$ the concavity is positive characterizing an extreme relativistic limit

\footnotetext{
${ }^{1}$ In fact any inertial frame can be considered with respect to which the velocities are constructed. The results of the numerical simulations and the related histograms will not change as can be verified.
} 


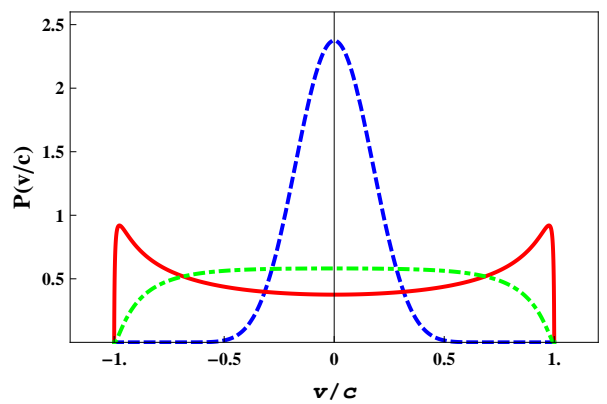

Figure 4: Plot of the 1D LI velocity distribution (8) for a fixed mass and decreasing values of the adimensional parameter $\beta$ (successively dashed, dashdotted and continuous curves, cf. text). The distribution is zero for $v^{2} / c^{2} \geq 1$, as expected.

for $\beta \ll 1$. On the opposite side for $\beta \gg 1$ we have approach the MB regime. For the Jütnner distribution (9) with $d=1$ the concavity at zero velocity changes for $m_{0} \beta_{J}=3$ showing that a high relativistic regime is attained at a lower temperature, as compared to the LI distribution (8).

We should finally remark that the variable $\sigma$ is in fact a particular case of the LI distance measure in the 3-dim Lobachevsky velocity space, which is the space of velocities in the special theory of relativity, as discussed in [5].

\section{Discussion on how to construct his- tograms of velocities for a relativistic gas}

In recent past years a large number of numerical simulations of a relativistic gas has appeared in the literature [6, 16, 11, 12, 13, 17. In these simulations the authors have studied some proposals of distribution of velocities for a relativistic gas, like the Jüttner distribution [10] or the Jüttner modified distribution [6]. The overall conclusion of all of these papers is that the Jüttner distribution matches accurately the data obtained by relativistic molecular dynamic simulations, convincing the majority of the scientists that Jüttner is the correct relativistic 
distribution. In the two following subsections we discuss some issues connected to the Lorentz invariance in these simulations and we distinguish approaches to construct the histograms of velocities to be compared with the theoretical distributions.

\subsection{Histograms with constant velocity bins}

In the molecular dynamical simulations used previously in the above cited papers, the histograms of velocities have been constructed using the event driven simulation method [7, 8]. After starting the simulations with the particles of the gas having random position and velocities and waiting the equilibration time, they collect the velocities of the particles at a precise moment and store them in a pool. The procedure is repeated many times and when the pool of velocities has a large number of data a histogram is constructed having in the abscissa equal size bins of difference of velocities. In our simulations here we have 100 samples, each with $N=5,000$ particles and equilibration time equal to $100 \times N$.

Let us discuss the construction of these histograms in one dimension. The extension for two or three dimensions is straightforward. In one dimension we divide - in the abscissa - the domain of the values of the velocities obtained, say $[-c, c]$, in $n$ intervals (bins) of equal size $(n=60)$. In the ordinate of the $j$-th bin $(j=1, \cdots, n)$ we put the number of all particles having, at the moment of the measurements, velocities comprised between the limits of the bin. The histogram thus obtained, after normalized, is compared with the theoretical relativistic distributions proposed in the literature, as the Jüttner distribution, that in $d$-dimension is given as

$$
f_{J}\left(v, m, \beta_{J}\right)=\frac{1}{Z_{J}} m_{0}^{d} \gamma(\mathbf{v})^{2+d} \exp \left[-\beta_{J} m_{0} \gamma(\mathbf{v})\right],
$$

where $m_{0}$ is the rest mass of the particles and $\beta_{J}=c^{2} / \kappa_{B} T$, where $\kappa_{B}$ is the Boltzmann constant. The one dimensional Jüttner distribution is obtained by simply taking $d=1$ in Eq. (9) 2 . Adopting this procedure, all the numerical simulations in the literature referred to here match accurately with the Jüttner distribution (cf. for instance [6] in 1-dim, [11, 12] in 2-dim and [13] in 3-dim). As a consequence

\footnotetext{
${ }^{2}$ In the 1-dim case it is necessary that a fraction of the $N$ particles considered in the simulations (let us say $N / 2$ ) have distinct masses in order that the distribution of initial velocities do not remain unaltered and that the system undergo equilibration [9, 6].
} 
of these results the present well-established position adopted by most of the scientists of the statistical mechanics community is that the correct relativistic distribution is the Jüttner distribution. A striking illustration of this can be seen in [6] (Figure 1), 11] (Figure 1) and [13. (Figure 1).

In our simulations we have also performed a 1-dim relativistic dynamical molecular simulation and the comparison of the histogram (constructed with constant velocity bins) with the Jütner distribution is displayed in Figures 1 and 2 (left) for heavy and light particles. As it can be seen, the agreement is also quite accurate.

However we consider that two points are not satisfactory within this approach. The first one is that the theoretical Jüttner distribution is not a LI distribution. In fact not only the relativistic energy, present in the argument of the exponential of Eq. (9), is not a LI quantity but also the correct $\gamma(\mathbf{v})$ factor needed to guarantee Lorentz invariance in 3D is $\gamma^{4}(\mathbf{v})$ (connected to the invariant element of volume of the Lobachevsky space of relativstic velocities) and not $\gamma^{5}(\mathbf{v})$ as it appears in the Jüttner distribution. The second point is that, in the construction of the histograms, in the abscissa, where the $\mu$-th bin (which initially is constant for any $\mu$ and equal to $\Delta=2 c / n$, for $n$ bins) corresponds to particles having velocities going from $v_{\mu-1}$ to $v_{\mu}$, is not LI, since the difference $\Delta_{\mu}=v_{\mu}-v_{\mu-1}$ (the size of the $\mu$-th bin) changes under a Lorentz transformation, i.e., all the bins in the abscissa change differently under a Lorentz transformation. Therefore, the diagram is not LI as well, presenting a different form in each reference frame. Consequently, we have the following (uncomfortable) situation: a histogram that is not LI matches well a distribution of velocities (Jüttner) that is not as well LI. This certainly is not a satisfactory theoretical framework. What we have to search is to construct a LI histogram of velocities and then try to find a LI distribution of velocities that matches it. This is the aim of our work.

We would like to remark that in a non-relativistic gas, whose particles obey the Maxwell-Boltzmann distribution, the whole system should be invariant with respect to a Galilean transformation of velocities. In a one dimension gas, for simplicity, let us consider the difference $\nu_{i}$ of velocities of the $i$-th particle $(i=1, \cdots, N)$ with the velocity of the box in a Galilean inertial frame, $\nu_{i} \equiv v_{i}-v_{\mathrm{box}}$. In the abscissa let us divide the domain of the values of the variable $\nu_{i}$ obtained in the simulations (say $\left.\left[-\nu_{\min }, \nu_{\max }\right] \subset(-\infty, \infty)\right)$ in $n$ intervals (bins) of equal size where the $\mu$-th bin $(\mu=1,2, \cdots n)$ is written 
$\Delta_{\mu}=\nu_{\mu}-\nu_{\mu-1}(\equiv \Delta$ for all $\mu)$, with $\nu_{0} \equiv-\nu_{\min }$ and $\nu_{\mu}=\mu \Delta+\nu_{0}$. The size of each bin is equal to $\left(\nu_{\min }+\nu_{\max }\right) / n$, where $\nu_{\min }$ is the maximum velocity obtained in direction $-x$ and $\nu_{\max }$ is the maximum velocity obtained in direction $+x$. We remark that $\Delta_{\mu}$, for any $\mu$, remains constant after a Galilean transformation. If in the ordinate we put in the $\mu$-th bin the number of particles whose associated value of $\nu_{i}=v_{i}-v_{\text {box }}$ is comprised between $\nu_{\mu-1}$ and $\nu_{\mu}$, the ordinate also does not change after a Galilean transformation. This implies that the whole histogram thus constructed is Galilean invariant and is well-fitted with a Gaussian centered at zero in any Galilean frame. This is the framework we want to reproduce in the relativistic case.

We also note that if, in the ordinate, we put in the $\mu$-th bin the number of particles having velocities comprised between $v_{\mu}$ and $v_{\mu-1}$ (not the difference of velocity of the particle and the velocity of the box as previously), after a Galilean transformation the histogram fits well with a Gaussian centered at a nonzero velocity, that is the velocity associated with the Galilean transformation. Therefore, the best way to construct a histogram that is Galilean invariant is considering the difference of velocities between the particles and the box. The histogram so constructed fits with a Gaussian centered at zero in any Galilean frame.

\subsection{LI histograms: constant rapidity bins}

In the relativistic molecular dynamics, we also use the event driven simulation method with the relativistic scattering rules among the particles. In order to construct a histogram that is relativistic, or Lorentz, invariant, following the scheme of the non-relativistic case (that is Galilean invariant), we want that nor the abscissa neither the ordinate of the histogram change after a Lorentz transformation. Clearly, the bins constituted by the difference of velocities $\Delta_{\mu}=\nu_{\mu}-$

$\nu_{\mu-1}$ are not LI. It is therefore crucial to use in the abscissa a quantity that is LI. To do this, let us first consider the relativistic difference $\mathfrak{v}_{i}$ between the velocity of the $i$-particle $\left(\mathbf{v}_{i}\right)$ and the velocity of the box $\left(\mathfrak{v}_{\text {box }}\right)$, given by (cf. (2) )

$$
\mathfrak{v}_{i}^{2}=\frac{\left(\mathbf{v}_{i}-\mathbf{v}_{b o x}\right)^{2}}{\left(1-\mathbf{v}_{i} \cdot \mathbf{v}_{b o x} / c^{2}\right)^{2}}
$$


We now consider the rapidity, $s_{i}$, of this difference, concerning the particle $i$, that is a LI quantity,

$$
s_{i}=\frac{1}{2} \ln \left(\frac{1+\mathfrak{v}_{i} / c}{1-\mathfrak{v}_{i} / c}\right)=\tanh ^{-1}\left(\mathfrak{v}_{i} / c\right) .
$$

We then propose to construct the abscissa of the histogram using the values of the rapidity, that now goes from minus to plus infinity. In the simulations we take the minimum and maximum numerical values obtained for the rapidity in all samples and divide this interval in the abscissa in equal rapidity-bins, where in the $\mu$-th rapidity-bin we put all particles having values of $\left\{s_{i}\right\}$ going from the inferior and superior limits of the bin. The normalized histogram constructed in this way is invariant by a Lorentz transformation, as expected, but it is a histogram of the rapidity. In order to get a histogram of the velocities, or of the difference of velocities between the particle and the box, we use the inverse transformations of Eqs. (11) and (10) (once we know the velocity of the box) on the abscissa. These transformations will change the relative size of the bins, since now the bins are no longer equally spaced in a velocity scale, as they are in a rapidity scale. However, even if we now represent the rapidity histogram in a velocity-scale, this histogram is still LI.

In Figure 3 we show the histogram in a scale of difference of velocities for $N=2,500$ light particles (the simulations have also $N=2,500$ heavy particles) obtained from the histogram of the rapidities for the light particles. The agreement of the numerical data with the best fit of the theoretical LI velocity distribution (8) is excellent (continuous line), with a normalized rms between the curve and the points $\simeq 0.008$. We also represent in Figure 3 the best-fit of the Jüttner distribution (dashed line) with the data of the histogram, where the parameter $m_{0} \beta_{J}$ has been adjusted. Clearly the Jüttner distribution does not fit the data, in contrast to the case where the histograms are constructed, from the beginning, with constant bins of velocities. This indicates that the Jüttner distribution cannot be considered as the relativistic partner of the Maxwell-Boltzmann distribution. We would like to remark that in our simulations we obtained, with the method of constant bins of velocities, a good agreement with the results of [6], matching the Juttner distribution. Our results were obtained from 100 simulations with $N=5,000$ particles, with a simulation time of $T=100 \times N$ and with a fixed number 60 of event points for the equilibrium configuration. The best fit of the Jüttner distributions yields 
values of the parameter $m_{0} \beta_{J}$ with a relative difference of only $3 \%$ for heavy and light particles (cf. Figs. 1,2 (left)). However, larger differences of the values of $\beta$ appear in the LI distributions and we are performing further simulations to completely clarify this point.

\section{Conclusions}

We have examined here the full relativistic molecular dynamics of a 1-dim gas, by basically reproducing the numerical simulations made in a landmark paper on the subject [6], where the authors established numerically that the Jüttner velocity distribution function is the correct generalization of Maxwell's velocity distribution in special relativity. We were led to look into this problem again since we considered such conclusion not satisfactory mainly due to the fact that the Jüttner distribution is not LI. We therefore approached this problem using the same numerical simulations but adopting two distinct procedures on constructing the histograms with the data obtained. The first one corresponds to the analysis of [6], where the histograms are constructed with equal size bins in the velocity variables and is - by construction - not LI. Using this procedure we were able to reproduce the results of [6], where the equilibrium distribution of the velocities resulting from the relativistic simulations fits accurately the Jüttner distribution. The second procedure, which is a new contribution of this paper, adopts histograms based on the rapidity, a variable which is LI and is associated with the relative velocity of the particles: the histograms are constructed with equal size bins in the rapidity and are LI in the same way that the histograms of Maxwell-Boltzmann distributions, constructed with equal size bins in the velocity, are Galilean invariant. From these rapidity histograms we get a histogram of the relative velocities, or of the difference of velocities between the particle and the box, by using the inverse transformations of Eqs. (11) and (10) (once we know the velocity of the box) on the abscissa. These transformations will change the relative size of the bins, since now the bins are no longer equally spaced in a velocity scale, as they are in a rapidity scale. However, even if we now represent the rapidity histogram in a velocity-scale, this histogram is still LI. We then show that the equilibrium distribution of velocities is accurately fitted by the LI velocity distribution (8) proposed in this paper but not by the Jüttner distribution. In this way we believe that the symmetric connection 
between the Maxwell-Boltzmann velocity distribution and the LI velocity distribution (8) is established, the first Galilean invariant and the second LI, by using the variable relative velocity with respect to a given inertial frame, provided that the evaluation of relative velocities involves respectively the "arithmetic addition" or the "Lobatchevsky addition".

\section{acknowledgments}

We are grateful to Prof. Constantino Tsallis for stimulating discussions and suggestions that were fundamental to the development of this paper. We also acknowledge the Brazilian scientific agencies CNPq, FAPERJ and CAPES for financial support.

\section{References}

[1] J. C. Maxwell, Philosophical Magazine XIX (1860) 19-32 and XX (1860) 21-37.

[2] L. Boltzmann, Wiener Berichte 66 (1872) 275-370.

[3] L. Boltzmann, Wiener Berichte 76 (1877) 373-435.

[4] R. Balian, From Microphysics to Macrophysics: Methods and Applications of Statistical Mechanics, Vol. I, Springer Verlag (Berlin, 1991).

[5] Evaldo Curado and Ivano Damião Soares, A LI velocity distribution for a relativistic gas, arXiv:1406.0777 (2014).

[6] D. Cubero, J. Casado-Pascual, J. Dunkel, P. Talkner and P. Hanggi, Phys. Rev. Lett. 99 (2007) 170601.

[7] M. P. Allen and D. J. Tildesley, Computer Simulations of Liquids (Clarendon Press, Oxford, 1991).

[8] J. M. Haile, Molecular Dynamics Simulations: Elementary Methods (Wiley, New York, 1992).

[9] J. Dunkel and P. Hänggi, Physica A 374, 559 (2007).

[10] F. Jüttner, Ann. Phys. (Leipzig) 18 (1911) 856.

[11] A. Montakhab, J. Casado-Pascual and M. Barati, Phys. Rev. E 79, 031124 (2009). 
[12] M. Ghodrat, A. Montakhab, Comp. Phys. Comm. 182 (2011) 1909.

[13] J. Dunkel,P. Hänggi and S. Hilbert, Nat. Phys, 5, 741 (2009).

[14] L. E. Reichl, A Modern Course in Statistical Mechanics, John Wiley \& Sons (New York, 1998).

[15] V. Fock, The Theory of Space, Time and Gravitation, Pergamon Press (Oxford, 1964), Chapter I, Sec. 17.

[16] F. Debbasch, Physica A 387 (2008) 2443.

[17] M. Mendoza, N. A. Araujo, S. Succi and H. J. Herrmann, Scientific Rep. 2 (2011) 611. 